\title{
UVOC-MAC: A MAC Protocol for Outdoor Ultraviolet Networks
}

\author{
Yiyang $\mathrm{Li}^{a}$, Jianxia Ning ${ }^{b}$, Zhengyuan $\mathrm{Xu}^{a}$, Srikanth V. Krishnamurthy ${ }^{b}$, Gang $\mathrm{Chen}^{a}$ \\ ${ }^{a}$ Dept. of Electrical Engineering, University of California, Riverside. \{yiyli,dxu,gachen\}@ee.ucr.edu \\ ${ }^{b}$ Dept. of Computer Science and Engineering, University of California, Riverside. \{jning, krish $\} @$ ee.ucr.edu
}

\begin{abstract}
As an alternative to radio-frequency (RF) communications, optical wireless communications (OWC) can support high data rates and low power operations while providing good jamming resistance. Our focus in this paper is on deep ultraviolet (UV) outdoor communications (UVOC) where solar blind and nonline-of-sight operations are attractive. Light beams from UV LED arrays serve as information carriers. In an abstract sense, this is similar to directional transmissions in RF; however, the PHY layer characteristics significantly differ due to atmospheric scattering. First, we perform extensive experiments on a UV testbed towards understanding signal propagation and the impact of the PHY on medium access. We find that UV propagation supports (a) fully duplex communications and (b) multiple data rate transmissions. Next, we propose a novel contention-based media access control (UVOC-MAC) protocol that inherently accounts for the UV PHY layer and fully exploits multi-fold spatial reuse opportunities. Evaluations via both simulations and analysis show that UVOCMAC effectively mitigates collisions and achieves high throughput. In particular, up to a 4-fold increase in throughput and $50 \%$ reduction in collision are possible compared to a MAC protocol agnostic to the UV PHY properties.
\end{abstract}

Index Terms-Optical wireless communications, UV, MAC

\section{INTRODUCTION}

Recently, unlicensed ultraviolet or UV (wavelength below 400nm) communications have been considered as an alternative to RF communications. Given the proliferation of wireless deployments in the ISM bands, the use of LED-based UV can be attractive for achieving low cost and high data rate operations. Meanwhile, communications in UV typically do not experience interference from commonly used RF systems and equipment. While intense solar energy introduces significant noise to infrared (IR) and visible light (VL) optical signals, UV-C (the deep UV band operating with wavelength 200$280 \mathrm{~nm}$ ) waves are immune to solar exposure [1]; this solar blind band makes corresponding UV systems the most attractive from among other bands. In addition, a desirable characteristic of UV communications is effective communications via nonline of sight (NLOS) links. When UV waves propagate, there are diverse paths from a transmitter to a receiver because of atmospheric scattering [1], [2], [3]. As long as certain number of photons sent by a transmitter arrive at a receiver (possibly via NLOS paths), a communication link can be established. All of the above factors make UV-C attractive for both rural/open and metropolitan/urban outdoor environments. Furthermore, UV$\mathrm{C}$ signals are inherently jamming-resistant and not easy to intercept (low probability of interception) at long distances due to their unique power decay profile. This makes UVC especially suitable for inter-aircraft communications or in tactical settings.

This work was supported in part by the ARO grant W911NF-09-1-0293, ARL grant DAAD19-01-2-0011, and NSF NeTS grant 0626912.
While there are some preliminary research efforts on UV communications, the design of higher layer protocols for UV has not received much attention. The properties of UV make the communication environment unique (as we show later); thus, these properties will have to be carefully considered when designing protocols. In this work, our objective is to design a MAC protocol for outdoor communications in the UV-C band.

Towards our goal, we first perform extensive experiments in UV-C band; we make the following observations: (1) NLOS links are likely to exist with various configurations ${ }^{1}$. (2) A transceiver can work in a full-duplex mode with certain configurations. (3) The channel delay spread is different with different configurations; thus the possible data rates will also differ. From the experimental data, we also derive an empirical path loss model for NLOS communications to capture the extent of signal attenuation.

Based on the PHY layer properties assessed by our experiments, we propose UVOC-MAC, a MAC protocol that is tightly dependent on UVOC PHY properties. UVOC-MAC fully exploits multi-fold spatial reuse opportunities with appropriate configurations.

More specifically, we make the following contributions:

- Experimentation on a UV testbed: We perform extensive experiments on an outdoor UV PHY testbed. We obtain insights on physical aspects that impact the MAC design. First, we find that due to NLOS links there can be multiple possible links (in different configurations) between a communicating pair. Second, different configurations result in different delay spreads, which in turn determine the achievable data rates. Third, full-duplex communications are feasible in some specific configurations; if appropriately invoked this can in essence double the capacity of the link.

- Design of UVOC-MAC: We design UVOC-MAC to account for the above factors. In a nutshell UVOC MAC effectively exploits NLOS communication links and fullduplex possibilities. It also mitigates some of the sideeffects that arise due to these functions (discussed later). It also alleviates problems such as deafness and hidden/exposed node problems, commonly encountered in directional RF communications.

- UVOC-MAC Evaluations: We provide a theoretical analysis and also perform simulations to validate the efficacy of designed UVOC-MAC protocol in delivering high throughput and low collision. The collision probability is decreased by about $50 \%$ as compared to a MAC that does not take the UV PHY properties into account. In some cases, the throughput can increase by up to 4 times

\footnotetext{
${ }^{1}$ A configuration refers to a combination of the transmission direction and pointing angle of the transceiver. We define these terms formally later.
} 
compared to a protocol that is agnostic of the UV-PHY.

The rest of the paper is structured as follows. In Section II, we describe the PHY characteristics of UVOC. In Section III, we present the UVOC-MAC design. In Sections IV and V, we evaluate the performance of UVOC-MAC via analysis and simulations, respectively. In Section VI we review directional MAC protocols for RF and discuss why they are inappropriate for UV. We conclude our work in Section VII.

\section{EXAMINING THE UV PHY}

In this section, we discuss three physical characteristics of UVOC that impact MAC design. They are (1) the existence of NLOS communication paths, (2) the impact of delay spread on the achievable data rates, and (3) the ability to establish full-duplex communications. We perform extensive experiments towards achieving our goals. We also derive an empirical noncoplanar path loss model for UV communications.

Experimental setup: The testbed used for experiments is shown in Fig. 1. In our experiments, we employ LEDs, a solar blind filter, and a photon detector in an open space. The equipment also has a Q-switched solid state UV laser which can be used as necessary instead of the LEDs. The transceivers employ energy-based modulation (e.g., pulse position modulation (PPM) or on-off keying (OOK)). At the receiver an energy detector is employed.

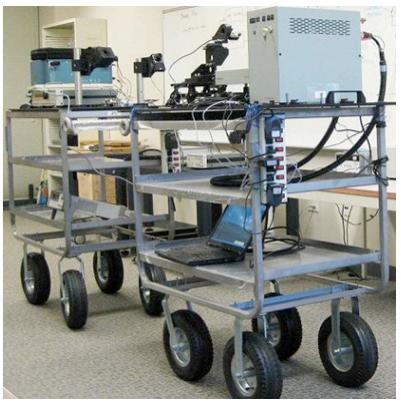

Fig. 1: The UV testbed. (Left cart: photon diode and oscillators; right cart: LED/laser transmitter)

NLOS communication paths: UVOC adheres to the commonly used signal-to-interference-plus-noise-ratio (SINR) model. In order to compute the SINR, one needs to characterize the attenuation that a UV signal experiences. Prior work on UV [1]-[3] models coplanar NLOS scattering path loss under the motivation of Reilly's common-volume single scattering theory [4]. From Reilly's theory, if there is a common volume (the overlap volume shown in Fig. 2) between the transmission beam and the receiver's field of view or FOV (see Fig. 3), the transmitted signal could be potentially detected. Thus, the transmitter and the receiver can successfully communicate not only via the LOS path but also via NLOS paths. NLOS links allow a node to have the choice of more than one configuration to communicate with a neighbor node. However, the increase in communication opportunities also results in increased interference in a transmitter's neighborhood. We analytically assess the tradeoffs in Section IV.

\begin{tabular}{|l|l|}
\hline The filter transmission efficiency & 0.1 \\
The PMT detection efficiency & 0.2 \\
LED power & $0.2 \mathrm{~mW}$ \\
Wavelength & $259 \mathrm{~nm}$ \\
Noise & 16 photons/s \\
Mismatch & 0.95 \\
Beam angle & $15^{\circ}$ \\
Field of view (FOV) & $30^{\circ}$ \\
Receiving pointing angle & $90^{\circ}$ \\
\hline
\end{tabular}

TABLE I: Experimental settings.

To date, there is no elegant path loss model for noncoplanar and multiple scattering UVOC. However, in a network setting, there is no guarantee of coplanarity. The coplanar path loss model presented in [3] requires the transmission beam axis and the receiver FOV axis lie in the same plane. The more recently proposed non-coplanar path loss model in [5] captures the effect of partial alignment (NLOS). Grounded in Reilly's theory, that model is however unable to capture the effects of multiple scattering which occurs in practice. In the following, we describe our empirical non-coplanar path loss model developed on the basis of experiments.

Computing path loss: First we measure the non-coplanar impact on path loss. The transmitter and receiver baseline separation is up to $50 \mathrm{~m}$. Using a curve-fitting method, a path loss model which depends on transmission pointing angle, offaxis angle and baseline separation is developed. The parameter settings are listed in Table I. The values of filter transmission efficiency and PMT detection efficiency are hardware parameters that affect the received energy. The LED power refers to that of a single LED; we use two LEDs (bound together) in all of our experiments. Given the LED power and actual receiving energy per counting interval, we can derive the path loss from the ratio. The background noise and device dark noise are negligible during experiment.

The beam angle defines the transmission full beamwidth [3]. Together with the receiver's FOV, it is shown in Fig. 3. We fix their values as in Table I.

Off-axis and pointing angles: The off-axis angle $\varphi$ is depicted in Fig. 4 and derived in Eq. (1). It specifies the horizontal deviation from the coplanar axis of two nodes. The vertical deviation from the coplanar axis is referred to as the pointing angle [5]. The transmitting angle $\alpha=\frac{\pi}{K}+n \frac{2 \pi}{K}(n=0,1,2, \ldots, K-$ $1) ; K$ is the total number of directions and in Fig. $4, K=4$. With fixed transmitter and the receiver coordinates, angle $\beta$ is determined (see Fig. 4).

$$
\begin{aligned}
& \gamma=(\pi-\alpha+\beta) \bmod 2 \pi \\
& \varphi= \begin{cases}\gamma & 0<\gamma<\pi \\
2 \pi-\gamma & \pi<\gamma<2 \pi\end{cases}
\end{aligned}
$$

UV non-coplanar path loss model: We leverage the following coplanar UV path loss model developed in [2]

$$
L_{\text {coplanar }}=\frac{p_{t}}{p_{r}}=\xi r^{a},
$$

where $L$ denotes the path loss, $p_{t}$ is the transmission power, $p_{r}$ is the received power at the detector, $r$ is the horizontal separation distance of the transmitter and receiver, $\xi$ is the path loss factor and $a$ is the path loss exponent. $\xi$ and $a$ are functions of the transmitter and receiver pointing angles [2].

If the off-axis angle between the transmitter and the receiver is non-zero, the received power decreases and the aforementioned coplanar UV path loss model is no longer valid. Our 


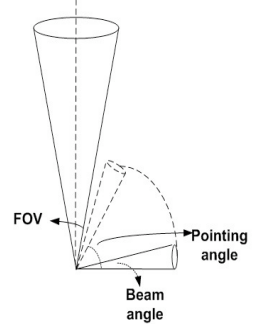

Fig. 3: FOV, beam angle and pointing angle

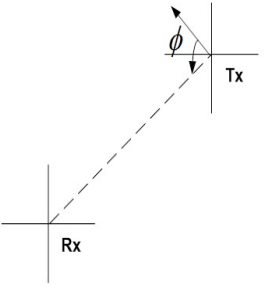

Fig. 4: An illustration of off-axis angle $\varphi$ from atop.

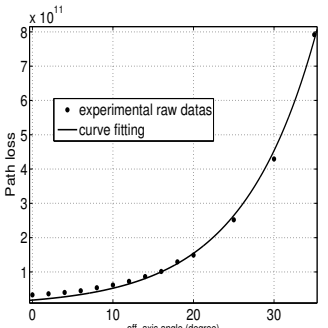

Fig. 5: Exponential curve fitting (pointing angle $=15^{\circ}$, distance $=15 \mathrm{~m}$ ).

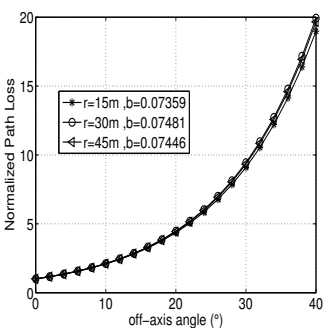

Fig. 6: The impact of distance on the exponent factor $b$ (pointing angle $\left.=30^{\circ}\right)$

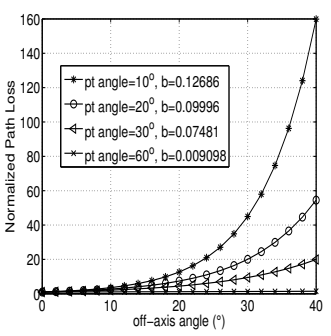

Fig. 7: The impact of the pointing angle on the exponent factor $b$ $($ distance $=30 \mathrm{~m})$. experimental results (see Fig. 5) suggest that the coplanar path loss model can be extended to account for such cases. We collect photon counts every second for 20 times at every $2^{\circ}$ off-axis angle increment until the signal is overwhelmed by the noise. Using the process of curve-fitting on the experimental raw data in Fig. 5, we see that the path loss increases almost exponentially with increasing off-axis angles and can be described by the following model:

$$
L_{\text {non-coplanar }}=\xi r^{a} \exp (b \varphi)
$$

where, the off-axis angle $\varphi$ is given by Eq. (1). The exponent factor $b$ is not (significantly) affected by varying distance $r$. Experiments show that $b$ is $0.07359,0.07481,0.07446$ for distances of $15 \mathrm{~m}, 30 \mathrm{~m}$ and $45 \mathrm{~m}$, respectively (See Fig. 6). But we observe that $b$ increases as the pointing angle decreases in Fig. 7. This is not surprising due to the common volume theory if single scattering is dominant; the overlapped volume shrinks rapidly with increasing off-axis angle at lower pointing angles. The path loss caused by off-axis angle increases faster when the pointing angle is smaller. To ensure SNR above certain threshold, the maximum tolerable off-axis angle varies with the pointing angle; it increases with increased pointing angle, and takes values of $5^{\circ}, 7^{\circ}, 15^{\circ}$ and $20^{\circ}$ with pointing angles of $10^{\circ}, 30^{\circ}, 40^{\circ}$ and $60^{\circ}$, respectively.

Spatial reuse with NLOS links: Nodes can increase spatial reuse by tuning the pointing and off-axis angles to create appropriate NLOS links. In Fig. 8(a), transmitter $T_{2}$ should not use pointing angle corresponding to the dark pointing angle to communicate with receiver $R_{2}$ in order to avoid interfering with the ongoing communication between $T_{1}$ and $R_{1}$. Instead, $T_{2}$ can choose the grey pointing angle to transmit. In the scenario in Fig. 8(b), tuning the pointing angle does not solve the collision problem with $T_{1} \rightarrow R_{1}$ (dark direction). $T_{2}$ should use a different off-axis angle (grey direction) to communicate with $R_{2}$.

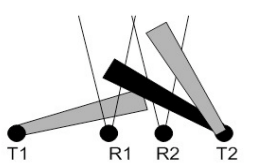

(a)

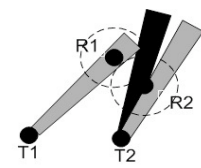

(b)
Fig. 8: NLOS links (a) front-view; (b) top-view. $\left(T_{1}, T_{2}\right.$ are transmitters and $R_{1}, R_{2}$, the corresponding receivers)
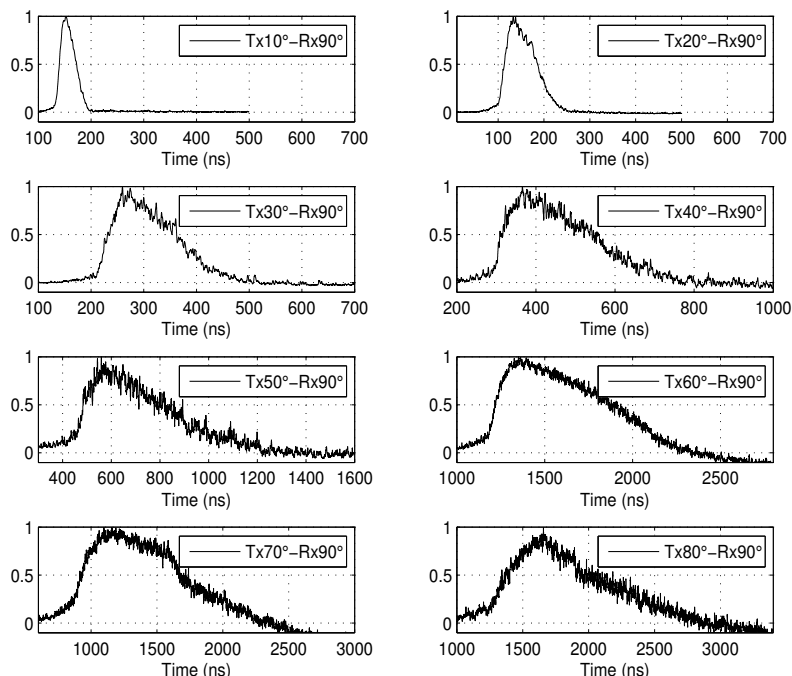

Fig. 9: Normalized impulse response with various transmission pointing angles in the coplanar case (the transmitter is $100 \mathrm{~m}$ apart from the receiver) [6].

Impact of delay spread: The impulse response characterizes the received energy temporal distribution. We observe that the the UV channel delay spread varies when the transmission pointing angle and off-axis angle are tuned. The measurement results in Fig. 9 show that delay spread increases with the pointing angle. This is because the common volume increases as the pointing angle increases (with other parameters fixed), and thus leads to addition of scattering paths of significant length, equivalently larger delay spread. We also see from Fig. 10 that the off-axis angle does not significantly impact delay spread; this is because the common volume does not change significantly when the off-axis angle is varied within certain range. We consider that one symbol is encoded per pulse and our objective is to eliminate inter-symbol interference or ISI (meaning that we wish to ensure that there is no overlap between consecutively received symbols). This would imply that the pulse width will differ for different point angles. Since the pulse width dictates the achievable data rate, the data rates will vary with pointing angles. Based on our measurements, we see that we can employ four data rates corresponding to four pointing angles. The data rates are $8 r, 4 r, 2 r$ and $r$ (lowest rate) when the pointing angle is at $10^{\circ}, 30^{\circ}, 40^{\circ}$, and $60^{\circ}$, respectively. Note that when the data rate is doubled, the slot 
size (or pulse width) is halved. This feature has to be accounted for when designing a MAC protocol. We also observe that first three data rates (with the corresponding pointing angles) support full-duplex communications (as seen later); the last pointing angle with the lowest rate can only use half-duplex communications.
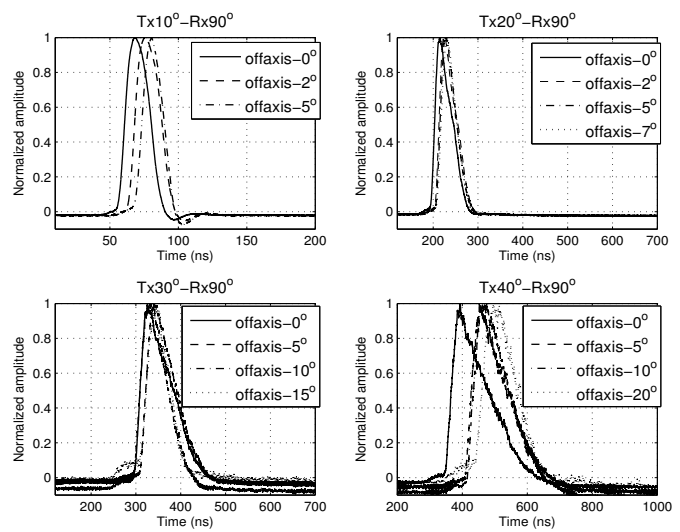

Fig. 10: Normalized impulse response with various off-axis angles (the transmitter is $50 \mathrm{~m}$ apart from the receiver).

Full-duplex communications: According to Reilly's common volume theory, simultaneous transmission and receptions at the same node is theoretically possible, as long as there is no intersections between its own transmission beam and its collocated receiver's FOV. As an example, with a $30^{\circ}$ FOV facing upwards and a $30^{\circ}$ beam angle facing the horizontal axis, full-duplex communications are achievable. In fact, it is easy to see that this is possible as long as the pointing angle is less than $60^{\circ}$ (see Fig. 3). We performed experiments to

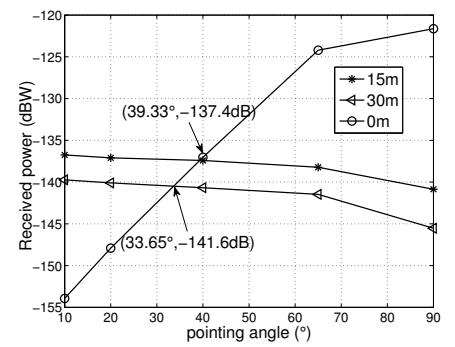

Fig. 11: Illustration of the feasibility of full-duplex.

test the feasibility of full-duplex communications in practice. The experimental data in terms of the received power versus the pointing angle is plotted in Fig. 11. We generally find that small pointing angles result in lower interference power to the collocated receiver and thus, are better suited for full-duplex communications. We observe that a pointing angle of about $40^{\circ}$ with a receiver transmission separation of $15 \mathrm{~m}$ (and 34 with a separation of $30 \mathrm{~m}$ ) can support decodable SIR. Larger pointing angles possibly result in overlapped volumes and cause increased interference level; thus, full-duplex communications are not feasible in these regimes.

In summary, small pointing angles can help achieve fullduplex communications and small delay spreads (as seen earlier). However, at these angles signals are more likely to be blocked by obstacles. Thus, a MAC protocol should opportunistically use small pointing angles, but at the same time, fall back to using larger angles as/when necessary.

\section{UVOC-MAC DESIGN}

In this section, we present our UVOC-MAC protocol. The design of UVOC-MAC is tied to the UV PHY properties: exploitation of NLOS links, the use of multiple rates and opportunistic use of full-duplex communications.

\section{A. Design of the UVOC transceiver}

Our system design is shown in Fig. 12. A similar design has been proposed for an indoor visible light communication system in [7]. The rationale behind our design is as follows.
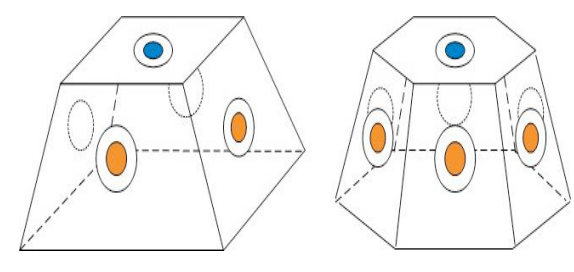

Fig. 12: System design of the UVOC transceiver with 4 (left) and 6 (right) transmission directions. (Dots on the side facets are the directional LED transmitters; dots on the top facet are the omni-directional photon receivers).

Since the photon detector is of high cost but the LEDs are of low cost, it is economical to implement multiple transmitters and a single receiver on a node. Given the dependencies between the pointing angle and full-duplex (introduced in Section II) communications, a good design will be for a node to have multiple surrounding transmitters and a receiver on top. This configuration provides favors to easy installation and deployment on the helmet of a soldier or on the top of a vehicle. In Fig. 12, the dots on the side facets represent the LED transmitters (a transmitter could consist of multiple simultaneously emitting LEDs) and the dot on the top facet represents the omni-directional photon detecting receiver. With this configuration, the node ${ }^{2}$ is a "directional transmitting" and an "omni-directional receiving" device.

Note that the real implementation is not limited to the shape or the number of facets. The number of directions or facets is determined by the transmitting beam angle. For example, if the beam angle of an LED array on a side facet is $18^{\circ}$, then this device can have up to 20 side facets. In other words, the number of directions is a system parameter that we can vary.

\section{B. UVOC MAC}

UVOC-MAC is a random access slot-based protocol. A single UV pulse is transmitted in each slot. The size of a generic slot is a multiple of what we call the unit slot size; the unit slot size corresponds to the highest data rate $8 r$.

The key feature of UVOC-MAC is that it exploits spatial reuse by adaptively choosing: (1) the direction, (2) the pointing angle and, (3) the data rate. Recall that the data rate is

\footnotetext{
${ }^{2}$ Without loss of generality, we also refer to a node as a transceiver.
} 
determined only by the delay spread which is different for different pointing angles, as discussed in Section II.

We first present a brief overview and then discuss operations of the protocol in detail.

UVOC-MAC Overview: In the default case, nodes are in the idle state where they simply decode received control signals. Once there is new data, a node first checks certain tables (discussed later) to see if communications are possible and if so, to decide on the best configuration for use. It then chooses a full-duplex mode or a half-duplex mode depending upon whether or not the selected pointing angle is less than or equal to $40^{\circ}$. Based on our experiments in Section II, we assume that with pointing angles less than or equal to $40^{\circ}$, full-duplex communications are possible; if the pointing angle is higher, then only half-duplex communications are possible.

The source and its destination exchange RTS and CTS messages at a base rate $r$ (we use similar nomenclature as in 802.11 for ease of presentation; however, the RTS and CTS messages are different here). The RTS message indicates the selected pointing angle; this also implicitly conveys the selected data rate (as discussed earlier, different data rates are possible with different pointing angles). Upon receiving the RTS, the receiver performs certain checks and replies with a CTS indicating the highest possible data rate (based on its interference patterns). The CTS is omni-directional by default and allows neighborhood nodes to update their neighborhood information. Then DATA is however sent directionally. There is a data rate announcement (DA) portion at the beginning of DATA frame. It is sent at the base rate and used to confirm the rate at which the remainder of the DATA is sent.

UVOC-MAC in detail: Next, we describe the UVOC-MAC in greater detail. We first describe a few data structures that are maintained for various purposes.

Connection table: Each node maintains a separate Connection table per neighbor. The table specifies the possible directions and pointing angles the node can use to communicate with this neighbor. The entries in the table are ranked in ascending order. There is more than one way to rank the directions and pointing angles. In Section V, we will compare two methods that can be used to rank entries in the Connection table. The space complexity of a Connection table is $O\{(\#$ of neighbors) · (\# of directions) · (\# of pointing angles) $\}$.

Occupancy table: This table is maintained at each node and is updated when the node realizes that a new transmission is going to take place in its neighborhood. To elaborate, when a CTS is received from a neighbor node $y$, a node first checks the Connection table associated with $y$. It then sets timers in the Occupancy table corresponding to those entries (an entry defines a combination of a direction and pointing angle) which define transmission configurations that would interfere with $y$ 's reception. The space complexity of this table is $O\{(\#$ of directions) . (\# of pointing angles) $\}$.

Rate table: This table stores a record of the data rates in use in the owner's neighborhood. As we discuss later, this information is used by the node to appropriately choose a transmission rate (pulse width) when it acts as a transmitter. The table is updated when the owner checks the first part of every directional DATA packet that it overhears; we refer to this part of the data packet as the "DA" (data rate announcement). The space complexity of this table is $O\{\#$ of pointing angles $\}$.
Receiving nodes table: This table enlists those neighbor nodes that are currently receivers and the time up to which they remain in the receiving mode. The contents of this table are disseminated using signaling messages and are used to combat effects such as deafness (explained later). The space complexity of this table is $O\{\#$ of neighbor nodes $\}$.

Next, we look at specific functions that a node invokes with UVOC-MAC.

Check table: A procedure check table is invoked in two cases: (a) when there is new data to send and (b) when a CTS is to be sent. In the first case, the source node checks the Connection table and Occupancy table to find the best combination of direction and pointing angle for use. If the best configuration (with the highest rank) is not available, it has to select an available configuration with a lower rank (it checks each possibility in descending order of rank). If a CTS message is to be sent in response to an RTS, the node checks the Occupancy table and chooses all available directions with the lowest available pointing angle.

Update tables: Every node has to update its aforementioned tables. The tables are updated with different stimuli and periods. The Connection table is constructed during the neighbor discovery process and updated by a neighbor maintenance procedure. Most neighbor discovery and neighbor maintenance schemes applied in networks with directional antennas (for e.g. [8], [9]) can also be applied with minor changes in UVOC networks; thus, we can simply leverage these approaches. In this paper, we assume that the neighbors of a node are known for simplicity. The Occupancy table and Receiving nodes table are updated upon receiving appropriate control signals and DATA packets. A node examines the source ID in a CTS message. It also checks the IDs of other receiving nodes from what we call the gossip portion (discussed later) in both the RTS and CTS messages. Finally, it checks the duration fields in the CTS, RTS and DATA messages. The Rate table is updated upon decoding the DA portion of every overheard DATA packet.

Gossip signaling: In order to mitigate the hidden node and deafness problems, nodes include information on receiving nodes in their neighborhoods, in the RTS and CTS messages. Note that only partial information can be sent with each message; only a subset of the nodes in the Receiving nodes table can be specified. There is a trade-off between the overhead incurred from the information (amount of information) and the efficacy in combating the aforementioned problems.

Frame structure: The frame structure with UVOC-MAC is shown in Fig. 13. The Duration field specifies the time duration of the communication. The Selected angle field specifies the pointing angle suggested by the receiver. The Time to wait field is used in relation to full-duplex communications. For instance, if node $A$ is sending RTS to $B$ and receiving from another node simultaneously, $A$ will experience a collision if $B$ sends the CTS right after receiving the RTS. Thus, $A$ informs $B$ using the Time to wait field, that it needs to wait for a certain time before sending the CTS. The Gossip signaling field contains information about neighborhood receivers as discussed earlier.

UVOC-MAC states of operation: The state transition diagram in Fig. 14 provides a complete depiction of UVOC-MAC.

In the Idle state, nodes simply decode received messages and update the corresponding tables. Upon receiving an RTS, a receiver checks the Time to wait field and sets a timer. It sends 


\begin{tabular}{|l|l|l|l|l|l|l|l|l|}
\hline $\begin{array}{l}\text { Frame } \\
\text { control }\end{array}$ & $\begin{array}{l}\text { Source and } \\
\text { direction ID }\end{array}$ & $\begin{array}{l}\text { Destination } \\
\text { ID }\end{array}$ & Duration & $\begin{array}{l}\text { Selected } \\
\text { Angle }\end{array}$ & $\begin{array}{l}\text { Frame } \\
\text { body }\end{array}$ & $\begin{array}{l}\text { Time } \\
\text { to wait }\end{array}$ & FCS & $\begin{array}{l}\text { Gossip } \\
\text { signaling }\end{array}$ \\
\hline
\end{tabular}

Fig. 13: MAC frame format

CTS upon timer reaching zero. The direction to send the CTS is determined by the Occupancy table. At this point, the node transits to the Receiving data state.

If a node has new data to send in Idle state, it runs the check table procedure and sends an RTS (directionally with the best available configuration) and transits state Waiting for CTS. In this state, a node still decodes signals that it receives and update tables if and until it experiences a CTS timeout. If the node receives the expected CTS, it enters the state Sending DATA; if not, upon timeout, it transits into state Backoff.

When a node receives a CTS it checks the Selected angle field to identify the direction of transmission and the corresponding rate. It then sends DATA directionally. While sending DATA, the node can receive other intended RTS messages depending on whether full-duplex communications are possible (the selected pointing angle is less than $40^{\circ}$ ). After having sent the DATA, the node stays in this state if it is still receiving from other nodes; otherwise it returns to the Idle state.

Handling problems with directionality: The use of an omnidirectional CTS transmission alleviates the problem of hidden nodes and deafness to a large extent. However, a receiver may sometimes have to use a directional CTS transmission since otherwise, it interferes with an ongoing communication. In such cases, these problems can arise. As discussed earlier, our gossip signaling successfully alleviates these problems to almost insignificant levels (as we observe with our simulations).

The exposed node problem is not an issue with UVOC-MAC since in most cases, full-duplex communications are possible.

The use of multiple-transmission rates: UVOC-MAC is slotted and adaptively employs four data rates denoted by $r$, $2 r, 4 r, 8 r$. The use of shorter pulse widths with PPM leads to higher data rates; recall that the choice of the pulse width is dictated by the delay spread which is in turn influenced by the pointing angle. The possibilities are shown in Fig. 15. A slot can accommodate two pulses. With binary PPM, a pulse in the first half of the slot (a sub-slot) indicates a ' 0 ' and a pulse in the second sub-slot indicates a ' 1 '. From the figure we see that when a high rate projects interference on a low rate, there is practically no impact. This is because the energy-based detector simply computes the difference in the energy between the two sub-slots of a slot and in each lower rate sub-slot, a similar interference energy is added. Note here, those results are for a given distance. As an example, when an $8 r$ rate transmission interferes with a $4 r$ rate transmission, an interference pulse is added to each sub-slot of the lower rate transmission. However, as one can immediately see, interference from lower rate transmissions impact higher rate transmissions. Thus, UVOC-MAC disallows lower rate transmissions if they project interference at the receiver of a higher rate transmission; it allows the initiation of lower rate transmissions as long as there is no such impact. This is the necessity of Rate table as well.

\section{ANALYZING COLlision PROBABILITIES}

Our goal in this section is to show that the collision probabilities in UVOC networks are high if no regulation is used (like with UVOC-MAC) via a simple analysis.

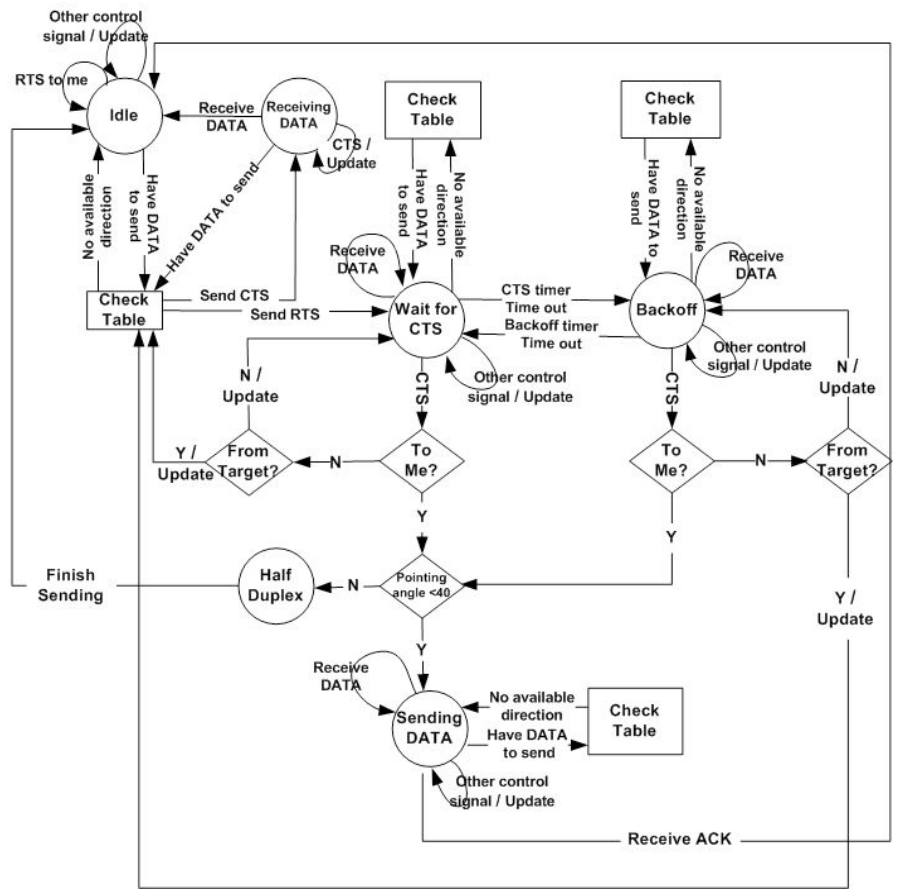

Fig. 14: Detailed state transition diagram of UVOC-MAC.

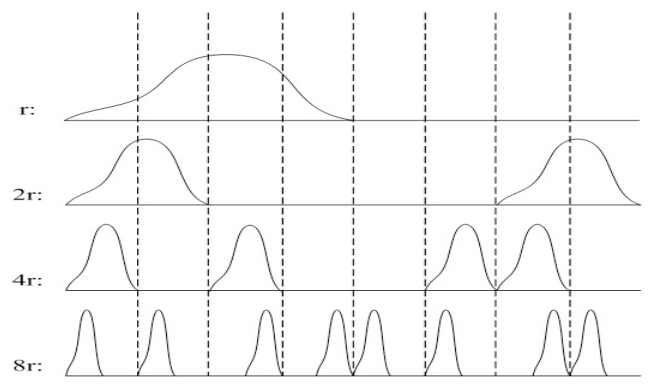

Fig. 15: Dependencies between different data rates.

Let us assume that the distance between the intended transmitter and receiver is $r_{0}$ and the corresponding off-axis angle is $\varphi_{0}$. The interferer (say $i$ ) is located at distances $r_{i}$ and has corresponding off-axis angles $\varphi_{i}$. We assume that interferers are uniformly distributed in the area with radius $R$ centered at the intended receiver. The $\varphi_{i}$ is uniformly distributed in $\{0, \pi\}$; the analysis can be easily modified for other distributions of $r_{i}$ and $\varphi_{i}$.

We consider two popular models for characterizing a successful reception: (a) the protocol model and (b) the physical model. With the two models, a collision occurs if:

$$
\operatorname{pr}(\text { collision })= \begin{cases}\operatorname{pr}\left(\max _{k}\left(p_{k}\right) \geq \frac{p_{0}}{T}\right) & \text { (protocol model }) \\ \operatorname{pr}\left(\sum_{k=1}^{m} p_{k} \geq \frac{p_{0}}{T}\right) & \text { (physical model })\end{cases}
$$

where $m$ is the total number of interfering nodes around the desired receiver, and $p_{k}$ is the received power from $k^{t h}$ interfering node.

Recall that with UVOC signal detection is typically energybased. Thus, the additive interference model is a good fit. In 
other words, the physical model can capture the behaviors with UVOC well. However, the protocol model offers simplicity and hence we consider it as well. With the protocol model we only consider the interference from the strongest interferer; thus, the performance with this model is somewhat optimistic.

The received signal power is given by $p_{0}=p_{t} c r_{0}^{-a} \exp \left(-b \varphi_{0}\right)$ (refer to Eq. (3)) and the interference power by $p_{i}=$ $p_{t} c r_{i}^{-a} \exp \left(-b \varphi_{i}\right)$. Here, the coefficient $c=1 / \xi$. Let $z=$ $r_{i}^{-a} \exp \left(-b \varphi_{i}\right)$. The probability density function (PDF) of $z$ can be easily computed given the PDFs of $r_{i}$ and $\varphi_{i}$ [10]. The pdf of $z$ is given by (we omit the derivation due to space constraints):

$$
f(z)= \begin{cases}\frac{1}{z b \pi}-\frac{\exp (-2 b \pi / a)}{b \pi R^{2}} \cdot z^{\left(-\frac{2}{a}-1\right)} & \left(\frac{\exp (-b \pi)}{R^{a}}<z<\frac{1}{R^{a}}\right) \\ \left(\frac{1}{b \pi R^{2}}-\frac{\exp (-2 b \pi / a)}{b \pi R^{2}}\right) \cdot z^{\left(-\frac{2}{a}-1\right)} & \left(\frac{1}{R^{a}}<z<\xi\right)\end{cases}
$$

Note here that path loss cannot be smaller than $1, z$ has an upper bound of $\xi$ so that PDF of $z$ needs normalization. We can then derive the probability that the interference power exceeds the desired threshold (as per the protocol model) to be:

$$
\begin{gathered}
P_{c}=\operatorname{pr}\left(p_{i} \geq \frac{p_{0}}{T}\right)=\operatorname{pr}\left(r_{i}^{-a} \exp \left(-b \varphi_{i}\right) \geq \frac{r_{0}^{-a} \exp \left(-b \varphi_{0}\right)}{T}\right) \\
=P\left(z \geq \frac{r_{0}^{-a} \exp \left(-b \varphi_{0}\right)}{T}\right) \\
=\left\{\begin{array}{r}
\frac{2 a \ln \frac{r_{0}}{R}+2 b \varphi_{0}+2 \ln T+a}{2 b \pi}-\frac{a}{2 b \pi} \frac{r_{0}^{2}}{R^{2}} \exp \left(\frac{-2 b \pi}{a}\right) \exp \left(\frac{-2 b \varphi_{0}}{a}\right) T^{\frac{2}{a}} \\
\text { when } \frac{r_{0}^{-a} \exp \left(-b \varphi_{0}\right)}{T} \leq \frac{1}{R^{a}} \\
\frac{a}{2 b \pi} \frac{r_{0}^{2}}{R^{2}}\left(1-\exp \left(\frac{-2 b \pi}{a}\right)\right) \exp \left(\frac{-2 b \varphi_{0}}{a}\right) T^{\frac{2}{a}} \\
\text { when } \frac{r_{0}^{-a} \exp \left(-b \varphi_{0}\right)}{T}>\frac{1}{R^{a}}
\end{array}\right.
\end{gathered}
$$

The final expression is simply obtained by integrating $f(z)$ over the desired interval. $P_{c}$ is the probability that one of the interferers (say $i$ ) causes a collision at a particular target. With the protocol model, a collision occurs if at least one of these interferers causes a collision. Thus,

$$
\operatorname{Pr}(\text { collision })=1-\left(1-P_{c}\right)^{m} .
$$

Numerical results based on the above analysis are compared with Monte Carlo simulation results using the proposed noncoplanar path loss model (referred to as the empirical model) and the single scattering non-coplanar path loss model from [5] (referred to as the SS model). With each, we consider both the protocol and physical collision models. $r_{0}$ and $\varphi_{0}$ are set to $67 \mathrm{~m}$ and $10^{\circ}$, respectively. $r_{i}$ is distributed with probability density $2 r / R^{2}$ in $\{0,100\} m$ an $\varphi_{i}$ is uniformly chosen from $\{0,180\}^{\circ}$. Note here that the mean value of $r_{i}$ is $67 \mathrm{~m} ; r_{0}$ is chosen to be this value. The comparisons are presented in Fig. 16. We observe that the analytical results match the results from the Monte Carlo simulations using the empirical model quite well. This is expected since the analysis uses the same path loss model as that of the empirical model. However, since the channel attenuation is different with the SS model, the results differ.

Second, we observe that the larger the number of interfering nodes, the larger the discrepancy between the analytical protocol model and empirical physical model. This is because as the number of interferers increases, the protocol model (accounts for only one dominant interferer) becomes less and less accurate.

Third, the discrepancy between protocol and physical models with the SS path loss model, is negligible. This is because the SS model inherently assumes that received power is zero if the interfering node has no common volume with the target FOV. With this, the accumulated interference is very close to the maximum interference. Recall however, this model is inaccurate as we see in our experiments.

Fourth, the SS model yields smaller collision probabilities than the empirical non-coplanar model as the SIR threshold increases. This is because, the multiple scattering is captured by the empirical model (Eq. (3)) and this accurately captures higher levels of interference thus, increased collision probabilities. In fact, due to this, the collision probability does not reach ' 1 ' for the range of SIR values considered.

Next, we consider the physical model in our analysis. It is extremely difficult to compute the distribution of $\sum_{k=1}^{m} p_{k}$. Thus, we use the central limit theorem [10] to approximate it as a Gaussian distribution. Towards this, we need to compute $E(z)$ and the variance $\operatorname{Var}(z)$. Since the pdf of $z$ is known, it is easy to compute these values and we provide them below.

$$
\begin{gathered}
E(z)=\frac{2[\exp (-b \pi)-1]}{\pi a b\left(1-\frac{2}{a}\right) R^{a}}+\frac{1-\exp \left(-\frac{2 b \pi}{a}\right)}{b \pi R^{2}\left(1-\frac{2}{a}\right)} \xi^{1-\frac{2}{a}}=\mu . \\
\operatorname{Var}(z)=\frac{\exp (-2 b \pi)-1}{2 \pi a b\left(1-\frac{2}{a}\right) R^{2 a}}+\frac{\xi^{2-\frac{2}{a}}}{2 b \pi R^{2}\left(1-\frac{1-\exp \left(-\frac{2 b \pi}{a}\right)}{a}\right)}=\sigma^{2} .
\end{gathered}
$$

With $\mu$ and $\sigma^{2}$ for $z$ computed as above, the collision probability can be expressed as

$$
\begin{aligned}
P_{c} & =\operatorname{pr}\left(\sum_{i=1}^{m} p_{i} \geq \frac{p_{0}}{T}\right)=\operatorname{pr}\left(S_{m}>A\right) \\
& =\operatorname{pr}\left(Z_{m}>\frac{A-m \mu}{\sqrt{m} \sigma}\right),
\end{aligned}
$$

where $Z_{m}=\frac{S_{m}-m \mu}{\sqrt{m} \sigma}$ and $m$ is the number of interferers. The results are shown in Figs. 17 and 18. From these two figures, we can see the CLT approximation holds well and there is a match with the Monte Carlo physical protocol results.

Multiple Rates: The above analysis (Eq. (10)) can be further extended to capture the impact of multiple rates. We can individually calculate the collision probability for transmissions at each data rate knowing that higher rate transmissions have no impact on the lower rate transmissions. For example, transmissions at data rate $r$ are only affected by other transmissions at rate $r$. On the other hand, transmissions at rate $8 r$ are affected by transmissions at all rates. Let $r_{1}$ denote $8 r, r_{2}$ denote $4 r$ and so forth. Let the $p\left(r_{1}\right), p\left(r_{2}\right)$ and so on be the probabilities with which interferers transmit with those rates. The corresponding expressions would be:

$$
p\left(\text { collision } \mid r_{j}\right)=p r\left(Z_{m}>\frac{A-\sum_{i=1}^{5-j} \mu_{i} p\left(r_{i}\right)}{\sqrt{\sum_{i=1}^{5-j} \sigma_{i}^{2}}}\right) .
$$

The collision probability averaged over the set of all possible data rates becomes

$$
p(\text { failure })=\sum_{i=1}^{4} p\left(\text { collision } \mid r_{i}\right) \cdot p\left(r_{i}\right) .
$$



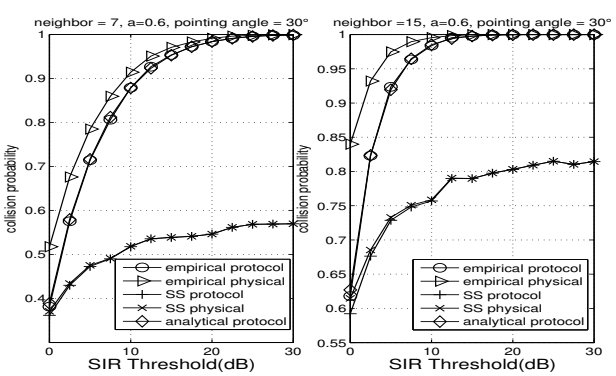

Fig. 16: Collision probability for a given pair of nodes with distance $r_{0}=67 \mathrm{~m}$ and off-axis angle $\varphi_{0}=10^{\circ}$ within the area $R=100 \mathrm{~m}$; the pointing angle is $30^{\circ}$.

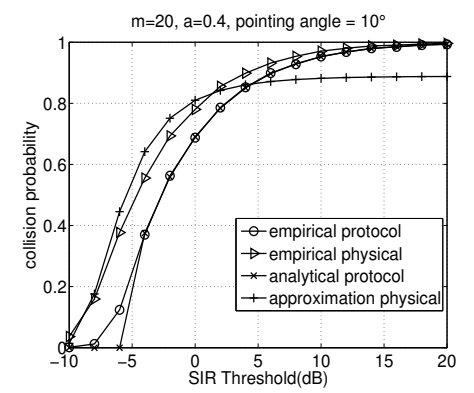

Fig. 17: Collision probability for a given pair with distance $r_{0}=67 \mathrm{~m}$ and off-axis angle $\varphi_{0}=10^{\circ}$.

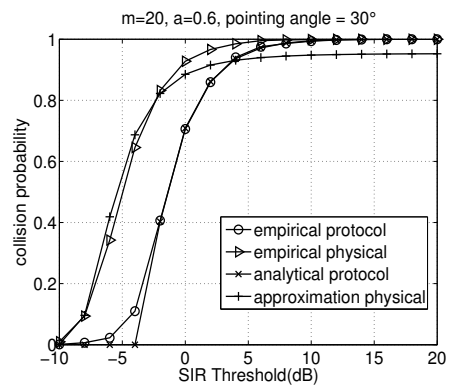

Fig. 18: Collision probability for a given pair of nodes with distance $r_{0}=67 \mathrm{~m}$ and off-axis angle $\varphi_{0}=30^{\circ}$.
The analytical results produced by Eq. (12) yield a collision probability of $70.59 \%$ if we were to use the distributions for $p\left(r_{j}\right)$ from simulation data when a node has 7 neighbors on average and the SIR threshold is $10 \mathrm{~dB}$. This extremely high collision probability is due to the lack of coordination in choosing directions and rates. Collisions occur both because of nodes transmitting to common receivers and also due to improper choice of rates. As we see in the following section, the use of UVOC-MAC drastically decreases this collision probability and provides significant throughput improvements.

\section{Simulation RESUlts}

In this section, we evaluate the performance of UVOC-MAC with OPNET version 16.0 [11].

Simulation settings: The default simulation settings are listed in Table II. The chosen transmission power corresponds to the typical short UV transmission range (approx 100m). A packet generated by a node is targeted towards a randomly chosen neighbor. We use the channel attenuation and delay spread results from our PHY experiments reported in Section II to characterize signal propagation.

\begin{tabular}{|l|l|}
\hline Transmission power & $4 \mathrm{~mW}$ \\
\# of directions & 6 \\
SIR threshold & $10 \mathrm{~dB}$ \\
Collision model & Physical (accumulative) model \\
Traffic pattern & $200 \mathrm{pkt} / \mathrm{sec}$ \\
Packet size & $1024 \mathrm{bits} / \mathrm{pkt}$ (data portion) \\
Network size & $100 \mathrm{~m} \mathrm{by} 100 \mathrm{~m}$ \\
\# of nodes & 8 \\
\hline
\end{tabular}

TABLE II: Simulation settings

Setting up and maintaining connection tables: Connection tables are assumed to be available upon neighbor discovery. There are two ways to rank the entries in Connection tables: (a) high-rate based method and path-loss oriented method. With the former, ranks favor lower pointing angles (thus higher rates). The entries associated with pointing angle leading to half-duplex communications are always ranked lower than those with smaller pointing angles. With the path-loss oriented method, ranks are assigned as per the measured path loss. Configurations with smaller path loss are assigned higher ranks.

Evaluating the ranking methods: With UVOC-MAC, we find that the above two maintenance methods result in different data rate usage distributions, throughput and collision probabilities. Naturally, the high-rate based method results in a more aggressive usage of high rates. The distributions with which the different data rates are used (from simulations) are shown in Table III. We see that $93 \%$ of the transmissions are at rate $8 r$. With the path-loss oriented method, in contrast, the rate $2 r$ is used by about $90 \%$ of the transmissions. The latter effect is due to the fact that lower pointing angles result in higher path loss for a fixed off-axis angle (seen in our experiments in Section II); thus, a lower path loss typically corresponds to a higher pointing angle and thus a lower rate. As seen from Fig. 19, the average throughput with the high-rate based method is about $74 \%$ higher than that with the path-loss oriented method. This is because higher rates directly lead to higher throughput.

In Fig. 20, we depict the usage of the different rates with varying node densities. The high-rate based method is used. We observe that as the node density increases, there is an increased requirement towards using higher pointing angles and correspondingly lower rates. This is because, spatial reuse is more difficult if only the highest rate is used; the pointing angle dimension will have to be more heavily utilized in such cases (higher pointing angle).

Collision probabilities with UVOC-MAC: We observe from simulations that the number of packets that are sent/received in a sample duration of 20 seconds. The results indicate that the collision probability (expressed as a percentage) is $19.83 \%$ with the high-rate based method while it is $23.61 \%$ when the path-loss oriented method is adopted. Note the drastic reductions that are achieved compared with $70.59 \%$ without UVOC-MAC (as discussed in Section IV).

\begin{tabular}{|l|l|l|l|l|}
\hline Rates & $r$ & $2 r$ & $4 r$ & $8 r$ \\
\hline High-rate based & 0 & 0 & $7 \%$ & $93 \%$ \\
\hline Path-loss oriented & 0 & $89.3 \%$ & $4.9 \%$ & $5.8 \%$ \\
\hline
\end{tabular}

TABLE III: Usage of different data rates.

Impact of tuning the pointing angle: One significant advantage of UVOC is the extra dimension of spatial reuse; as discussed this is exploited by varying the pointing angle. The additional spatial reuse from exploiting the pointing angle can be expected to lead to higher throughput. We conduct four sets of simulations to quantify the throughput improvements due to varying the pointing angle. The first set of simulations uses UVOC-MAC. In the other three sets, the pointing angles are fixed at $10^{\circ}, 30^{\circ}$ and $40^{\circ}$. Thus, adaptivity in the additional dimension is eliminated. From Fig. 21, we see that the usage of multiple pointing angles brings about a 10\% throughput increase compared to a case with a fixed $10^{\circ}$ pointing angle. 


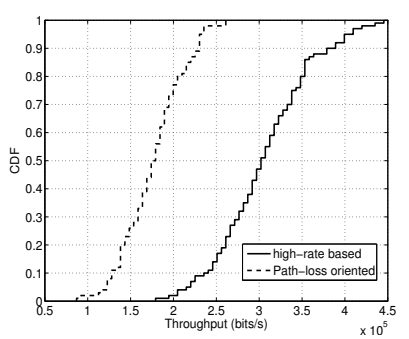

Fig. 19: Throughput comparison with the two ranking methods.

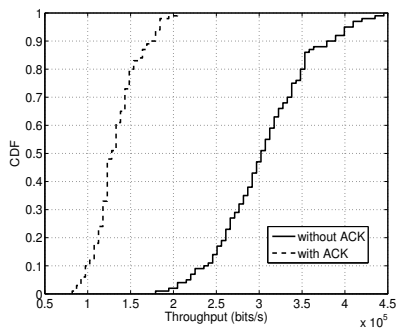

Fig. 23: ACK impact on throughput.
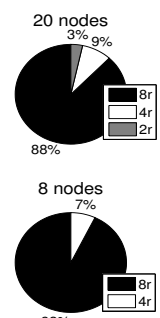

Fig. 20: Usage of the different data rates with node density.

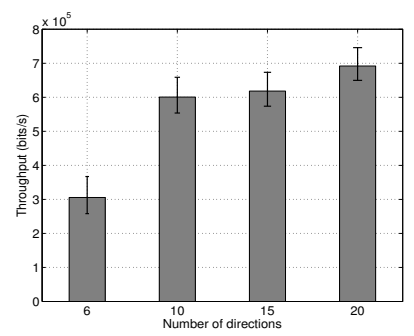

Fig. 24: Throughput with different numbers of directions.
Compared with the other two cases, the throughput gains are much higher. The throughput is about 4.3 times that with fixed pointing angles of 30 or $40^{\circ}$. These results first demonstrate lower pointing angles are better (as one might expect). More importantly, if pointing angles were to be statically assigned, it may turn out that some pairs would have to use higher pointing angles (especially in dense scenarios). This would drastically hurt their throughput. UVOC-MAC provides adaptive tuning to opportunistically exploit the usage of lower pointing angles which in turn, improves throughput. Therefore, we can conclude that due to the varying pointing angle, UVOC networks bear large spatial reuse opportunities and are capable of producing high throughput.

Exploiting full-duplex communications: Recall that UVOC nodes can communicate in full-duplex mode with small pointing angles. As discussed in Section II, a conclusion drawn from the experiments was that pointing angles lower than $40^{\circ}$ support full-duplex communications. We perform two sets of simulations to quantify the throughput gains from full-duplex opportunities with UVOC-MAC. In the first set, nodes can switch between half-duplex mode and full-duplex modes as per UVOC-MAC. In the other set, nodes operate in the traditional half-duplex mode. Fig. 22 depicts the throughput from the two sets. A $377.26 \%$ increase in throughput brought by full-duplex opportunities. Again, note that in dense settings, if pointing angles were statically assigned, certain pairs may be forced to just operate in half-duplex modes and thus, achieve much lower throughput than what is possible with UVOC-MAC.

ACK transmissions: We observe from simulations that if we transmit ACKs at the base rate with UVOC, collisions might occur during the ACK exchange period. This is primarily because these low rate transmissions affect other high rate transmissions in the node's vicinity. Since nodes already have prior rough knowledge about communications in their neighborhood from updates, we seek to examine if ACKs are indeed needed. Stated

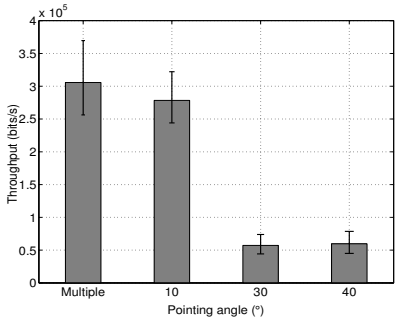

Fig. 21: Impact of pointing angle on throughput.

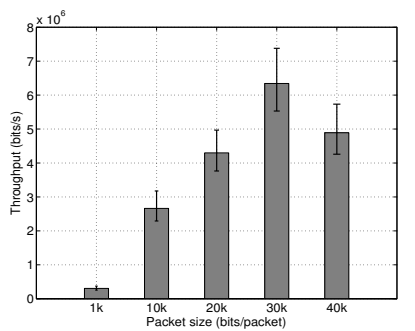

Fig. 25: Throughput with different packet sizes.

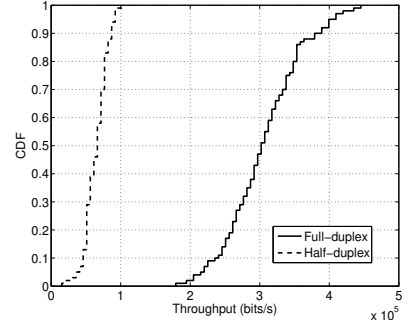

Fig. 22: Throughput benefits of full-duplex communications.

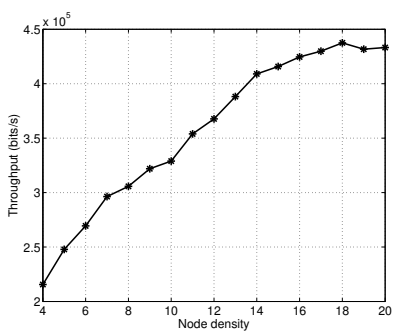

Fig. 26: Throughput with different node densities. otherwise, we perform simulation evaluations to understand if ACKs indeed help in improving throughput. Fig. 23 depicts the performance with or without ACK the process. We see that ACK procedure brings down the throughput by about $132 \%$; this loss is mainly due to the overhead and collisions with high rate transmissions. We conclude that it is better for UVOC to not include ACKs.

Impact of gossip signaling: Gossip signaling increases the awareness with regard to ongoing communications in a node's neighborhood. This can significantly reduce unnecessary RTSs that would interrupt other transmissions and/or fail to establish connections. We evaluate the impact of gossip signalling in a 20-node topology. From the simulation results we observe that the gossip procedure reduces the number of RTS messages initiated by $32.3 \%$. These are those RTS messages that did not result in a successful connection establishment.

Impact of system parameters: Finally, we examine the impact of various system parameters on the performance of UVOC-MAC.

Number of directions: Generally, the more the number of directions the better the spatial reuse. We find that UVOC-MAC effectively exploits an increase in the number of directions. From Fig. 24, we observe the throughput improves by $134.39 \%$ as the number of directions increases from 6 to 20 .

Packet size: Using longer packet sizes can potentially increase throughput due to decrease in the overhead per packet. However, it could also increase the collision probabilities. Fig. 25 shows the throughput trend with increasing packet size. To begin with, increasing packet size brings higher throughput. However, when the packet size increases to beyond a certain extent, the interference dominates and the throughput falls. The collision probability is $62.83 \%$ with $40 \mathrm{k}$ bits/packet compared with $19.83 \%$ for $1 \mathrm{k}$ bits/packet.

Node density: To examine the impact of node density, we vary the number of nodes from 4 to 20 . The nodes are uniformly 
distributed in a $100 \mathrm{~m}$ by $100 \mathrm{~m}$ area. Fig. 26 shows the network throughput versus node density. We see that the throughput is higher as node density increases. When the network is extremely dense $(\approx 20$ nodes) the throughput slightly decreases due to increased collisions. These results indicate that UVOCMAC maintains high throughput even with node densities.

\section{RELATED WORK}

We review some related work in the literature. Note that there has been little work done on higher layer protocol design for optical wireless communications, particularly in UVOC.

MAC protocols for $\boldsymbol{O W C}$ : The IEEE 802.11 standard [12] proposes a carrier sense based MAC scheme for indoor IR with LOS communication links. The MAC design in [13] suggests the usage of CSMA/CA in indoor IR ad hoc networks. [14] proposes a new linear contention window selection method, again for IR indoor networks. [15] suggests that TDMA-based MAC protocols could lead to the seamless integration of optical and RF segments. Most recently, a TDMA based MAC is proposed in [16] for an indoor IR infrastructure network.

The IEEE visible light communications (VLC) task group [7] is working on MAC design for indoor and vehicle-to-vehicle communications using visible light. They view the networks as WPANs whose existing protocols are modified to meet some emerging needs.

There is very limited work in the literature on outdoor OWC MAC. The aforementioned work is primarily on IR or VL transmissions in indoor environments. The infrastructure MACs are not suitable in ad hoc outdoor scenarios or in battlefields Most importantly, the existing approaches are based on carrier sensing; given the modulation strategies and channel asymmetry that are not appropriate for UVOC.

Directional MAC protocols in $R F$ : Given the narrow beam angle of wireless optics, the communications are directional. There exist many MAC protocols for use with directional antennas in RF [17]-[24]. The natural question that arises is whether these protocols can be directly applied in our considered context (although they are designed for RF). Due to the nature of UVOC, they are not directly applicable. First, physical carrier sense is not possible with UVOC. Second, they consider spatial reuse only in directions; here, we consider additional dimensions. DMAC [17] uses directional RTS/CTS to increase communication range, but suffers from deafness. Tone-DMAC [18] uses out-of-band signaling to solve the deafness problem. Out of band signaling requires the use of a different wavelength in OWC. In optics, switching across different wavelengths requires expensive filters and low-speed mechanical operation; further, it is difficult to implement multiple filters in a small portable device. Protocols such as [19] also use carrier sensing. CRM, CRCM, MDA and DMAC/DA [20], [21], [23], [24] use circular RTS/CTS to avoid the coverage asymmetry problem. A circular RTS/CTS is unnecessary in the context considered. A transmitter can simply turn on all LEDs in all directions at the same time without causing the asymmetry problem.

It can be observed that none of the existing protocols addresses non-line-of-sight scattering based optical transmission and reception, and our UVOC-MAC particularly targets such application scenarios.

\section{CONCLUSIONS}

Communications with UV light is a good alternative to RF in many scenarios. Higher layer design for UV networks will have to conform to UV PHY properties. In this paper, we first perform extensive experiments to understand and characterize the UV PHY layer. Based on the understanding, we design UVOC-MAC, a MAC protocol that inherently accounts for the UV properties. We perform analysis and simulations to demonstrate that UVOC-MAC effectively exploits spatial reuse in extra dimensions. The throughput can improve by up to 4 times compared to a MAC that does not adaptively exploit the properties of the environment.

\section{REFERENCES}

[1] Z. Xu and B.M. Sadler. Ultraviolet communications: potential and stateof-the-art. IEEE Commun. Magazine, 2008.

[2] G. Chen, Z. Xu, H. Ding, and B.M. Sadler. Path loss modeling and performance trade-off study for short-range none-line-of-sight Ultraviolet communication. Optics Express, 17(5), 2009.

[3] Z. Xu, H. Ding, B.M. Sadler, and G. Chen. Analytical performance study of solar blind non-line-of-sight ultraviolet short-range communication links. Optics Letters, 33(16), 2008.

[4] D.M. Reilly. Atmospheric optical communications in the middle ultraviolet. M.S. Thesis, MIT, Cambridge, MA, 1976.

[5] L. Wang, Z. Xu, and B.M. Sadler. Non-line-of-sight Ultraviolet link loss in noncoplanar geometry. Optics Letters, 35(8), 2010.

[6] G. Chen, Z. Xu, and B.M. Sadler. Experimental demonstration of ultraviolet pulse broadening in short-range non-line-of-sight communicaiton channels. Optics Express, 2010.

[7] IEEE 802.15 WPAN Task Group 7 (TG7). Visible Light Communication. http://www.ieee802.org/15/pub/TG7.html.

[8] S. Vasudevan, J. Kurose, and D. Towsley. On neighbor discovery in wireless networks with directional antennas. In IEEE INFOCOM, Mar. 2005.

[9] G. Pei, M. Albuquerque, J. Kim, D. Nast, and P. Norris. A neighbor discovery protocol for directional antenna networks. In MILCOM, Oct. 2005.

[10] Athanasios Papoulis. Probability, Random Variables, and Stochastic Processes. McGraw-Hill, New York, 1991.

[11] Opnet User's Documentation. http://www.opnet.com.

[12] IEEE Std 802.11 2007. Wireless LAN medium access control (MAC) and physical layer (PHY) specifications. http://standards.ieee.org/getieee802/download/802.11-2007.pdf.

[13] J.M. Kahn and J.R. Barry. Wireless infrared communications. In Proc. of the IEEE, 85(2), 1997.

[14] V. Vitsas and A.C. Boucouvalas. Performance analysis of the advanced infrared CSMA/CA MAC protocol for wireless LANs. Wireless Coтmunications, 9(5), 2003.

[15] P. Chowdhury, B. Mukherjee, S. Sarkar, G. Kramer, and S. Dixit. Hybrid Wireless-optical Broadband Access Network(WOBAN): Prototype Development and Research Challenges. IEEE Network, 23(3), 2008.

[16] N. Agrawal, S.D. Milner, and C.C. Davis. Free space optical sensor network for fixed infrastructure sensing. In SPIE, 2009.

[17] R.R. Choudhury, X. Yang, R. Ramanathan, and N.H. Vaidya. Using directional antennas for medium access control in ad hoc networks. In MobiCom, 2002.

[18] R.R. Choudhury and N.H. Vaidya. Deafness: A MAC problem in ad hoc netwroks when using directional antennas. In ICNP, 2004.

[19] A. Nasipuri, S. Ye, J. You, and R.E. Hiromoto. A MAC protocol for mobile ad hoc networks using directional antennas. IEEE WCNC, 2000.

[20] C.M. Cordeiro, H. Gossain, and D.P. Agrawal. A directional antenna medium access control protocol for wireless ad hoc networks. In Brazilian Telecommunication Society, 2005.

[21] T. Korakis, G. Jakllari, and L. Tassiulas. A MAC protocol for full exploitation of directional antennas in ad-hoc wireless networks. In MobiHoc, 2003.

[22] J. Wang, Y. Fang, and D. Wu. SYN-DMAC: A directional MAC protocol for ad hoc networks with synchronization. In MILCOM, 2005.

[23] H. Gossain, C. Cordeiro, and D.P. Agrawal. MDA: An efficient directional MAC scheme for wireless ad hoc networks. In GLOBECOM, 2005.

[24] M.Takata, M. Bandai, and T. Watanabe. A directional MAC protocol with deafness avoidance in ad hoc networks. IEICE trans on Communications, E90-B(4), 2007. 